\title{
An Application of Chance-Constrained Model Predictive Control to Inventory Management in Hospitalary Pharmacy
}

\author{
J. M. Maestre, P. Velarde, I. Jurado, C. Ocampo-Martínez, I. Fernández, B. Isla Tejera and J. R. del Prado
}

\begin{abstract}
Inventory management is one of the main tasks that the pharmacy department has to carry out in a hospital. It is a complex problem that requires to establish a tradeoff between different and contradictory optimization criteria. The complexity of the problem is increased due to the constraints that naturally arise in this type of applications. In this paper, which corresponds to preliminary works performed to implement advanced control techniques for pharmacy management in two Spanish hospitals, we propose and assess chanceconstrained model predictive control (CC-MPC) as a mean to relieve this issue.
\end{abstract}

\section{INTRODUCTION}

Inventory control is a classical problem that arises in many fields. Wherever there is an organization that provides a certain good or service, there is a need of controlling the items that are bought to this end. Ideally, the organization would know exactly when these items will be needed, and hence they could be ordered to arrive and be used just in time. Unfortunately, this is not realistic due to the existing uncertainties with respect to the demand and material or information delays. As a consequence, some conservatism in the control policy used is necessary in order to avoid stockouts, specially because the consequences of such event can be fatal.

Failures in the stock management in a hospital pharmacy may have catastrophic social and economical consequences. On the one hand, the clinical needs of the hospital have to be satisfied; the social cost of the unavailability of medicines may be enormous as it may lead to the loss of human lives. On the other hand, it is not possible to raise the average stock levels too much. Hospitals have tight budgets that impose constraints on the stock management. In [1] it is estimated that about $35 \%$ of hospital expenses on services and goods are due to the pharmacy department. In European countries, where the health care system is public, these expenses are

The authors would like to acknowledge Junta de Andalucía (Pharmacontrol Project, P12-TIC-2400), for funding this work.

J. M. Maestre, P. Velarde and I. Jurado are with the Departamento de Ingeniería de Sistemas y Automática Universidad de Sevilla, 41092 Sevilla, Spain. Emails: \{pepemaestre, pabvelrue, ijurado\}@us.es.

C. Ocampo-Martínez, Senior Member IEEE, is with Automatic Control Department, Universidad Politécnica de Catalunya, Institut de Robòtica i Informàtica Industrial (CSIC-UPC), Barcelona, Spain e-mail: cocampodiri.upc.edu

I. Fernández is with the Pharmacy Department, Hospital San Juan de Diós, Córdoba, Spain e-mail: MariaIsabel.Fernandez@sjd.es

B. Isla Tejera, J. R. del Prado are with Pharmacy Department, Hospital Universitario Reina Sofía, Córdoba, Spain e-mail: beatrizislatjegmail.com, joser.prado.sspal juntadeandalucia.es. millionaire. Therefore, inventory management is one of the main tasks that a pharmacy department has to carry out in a hospital. It is a complex problem because it requires to establish a tradeoff between contradictory optimization criteria. In addition, other factors that typically complicate inventory management problems have also to be taken into account in this context. For example, there are constraints on the placement of stocked drugs. Room is not endless, specially for those drugs that have to be preserved at low temperature, and thus have to be stored in a fridge. Delays on drug deliveries and non deterministic demands are also major issues in this context.

Typically, the pharmacy managers apply very simple inventory control policies. In particular, an $(s, S)$ policy is usually used, which means that when inventory drops below level $s$ an order is placed to raise it back to the inventory higher level $S$. Alternatively, a fixed size $Q$ can be assigned to the orders and then $s$ is defined as the reorder point. Note that other periodic review inventory control are possible as well, see for example [12] or [2]. Nevertheless, these policies lack of enough flexibility to take into account all the factors involved in this optimization problem in a systematic manner. For this reason, in this work we propose to apply model predictive control (MPC) to the pharmacy department inventory management problem. MPC is a popular control strategy for the design of high performance model-based process control systems because of its ability to handle multivariable interactions, constraints on control (manipulated) inputs and system states, and optimization requirements in a systematic manner. MPC takes advantage of a system model to predict its future evolution starting from the current system state along a given prediction horizon. Due to its high versatility, MPC has become one of the most popular control techniques in industrial applications [3]. In fact, similar problems such as supply chain management have also benefited from the application of MPC. For example, [13] and [14] applied MPC to supply chain management in semiconductor manufacturing. In [7] a popular supply chain benchmark, the MIT Beer Game, is used to test a distributed MPC algorithm with low communicational burden. Likewise, in [11] robust MPC is applied to production-inventory system. Finally, in [9] a variation of MPC is used to reduce the number of tuning parameters when managing inventories and supply chains.

In the design of predictive controllers for dynamical systems subject to disturbances and/or uncertainty, it is well known that even if the controller finds a feasible solution, there is a certain probability that real outputs may violate the system constraints. Therefore, it would be suitable to replace 
and/or reformulate the original constraints involving random variables by probabilistic statements, allowing not only the treatment of the uncertainty but also avoiding possible unfeasibility of the optimization problem behind the predictive controller. Probabilistic or chance constraints, which have been treated and developed within the stochastic programming framework [6], were firstly introduced during the 60 s 70s [8]. Combined with the standard MPC theory, they allow the designer to arise with a stochastic optimization problem behind the controller by replacing hard constraints (either of states or inputs) with probabilistic constraints and by replacing the nominal cost function with its expected value in the MPC formulation [4]. This stochastic approach, known as Chance-Constrained MPC (CC-MPC) demonstrates to be suitable for large-scale complex systems due to its inherent features such as robustness, flexibility, low computational requirements, and ability to include the level of reliability (or risk) associated with the constraints (which implies its a priori knowledge) [10], [5]. Thus, CC-MPC avoids the conservative nature of other MPC approaches taking into account the expected performance of the closed loop with proper constraint handling instead of directly trying to assure robust stability.

In this work, which has been performed in collaboration with two hospitals in Spain, we assess the use of CC-MPC to inventory management in Hospitalary Pharmacy, and it is a preliminary work of the project Pharmacontrol ${ }^{1}$. The goal of this collaboration is to update the inventory management system of these hospitals so it is possible to reduce the average inventory while maintaining the same clinical guarantees. In order to illustrate the size of the problem, we will say that the biggest hospital that participates in this work has a total capacity of 1200 beds for the inpatients. Besides these inpatients, the pharmacy department provides monthly more than five thousand drug dispensations for external patients. In this hospital the expenses on drugs exceed the amount of fifty millions of euros per year.

The paper is organized as follows. First, a description of the inventory management problem is shown in Section II. Section III, presents the optimization problem and the MPC for this problem. Section IV some simulations are shown. Finally, in Section V some conclusions are presented.

\section{Pharmacy InVentory Problem}

In this paper we assume that the pharmacy inventory is composed of $N_{i}$ different drugs. The following discrete linear model will be used to represent the evolution of the stock level of drug $i$ :

$$
s_{i}(t+1)=s_{i}(t)+\sum_{j=1}^{n_{p_{i}}} o_{i}^{j}\left(t-\tau_{i}^{j}\right)-d_{i}(t),
$$

where $s_{i} \in \mathbb{R}$ is the stock of $\operatorname{drug} i, o_{i}^{j} \in \mathbb{R}$ is the number of ordered items to the $j$-th of the $n_{p_{i}}$ providers of the drug $i, \tau_{i}^{j}$

\footnotetext{
${ }^{1}$ The project Pharmacontrol has as main objective the improvement of the stock control in hospital pharmacies, trying to reduce the stock mean level and, therefore, achieving considerable savings for the hospitals.
}

is its corresponding transport delay, and $d_{i}(k)$ stands for the aggregate demand of drug $i$. Note that the number of ordered items can be decomposed as $o_{i}^{j}=\delta_{i}^{j}\left(t-\tau_{i}^{j}\right) u_{i}^{j}\left(t-\tau_{i}^{j}\right)$, with $\delta_{i}^{j}(t)$ being a boolean variable whose value is one only if an order of drug $i$ to provider $j$ is placed during time $t-$ otherwise its value is zero - and $u_{i}^{j} \in \mathbb{R}$ being the actual number of ordered items in case an order is placed. This decomposition is introduced to simplify accounting for costs that are related to the placement of orders.

We consider the following costs associated to the inventory management problem:

- $p_{i}^{j}$ is the price that the $j$-th provider offers for drug $i$. This price could depend in general on the number of ordered items. On way to proceed in that case would be to estimate that dependency (adjusting with an expression that could be linear, quadratic, ...) to include explicitly in this term the decision variable $o_{i}^{j}$. We will assume for simplicity that this price does not depend on the number of ordered items.

- $C_{s h, i}^{j}$ is the shipping costs of asking drug $i$ to provider $j$.

- $C_{o p, i}$ represent the costs associated to placing an order of drug $i$.

- $C_{o s, i}$ is the cost of running out of stock of drug $i$, that is, the cost of shortage. In this case it is possible to ask for help to other hospitals. These loans require to contract special deliveries, which may have a high cost. In addition, the risk of not being able to satisfy the clinical needs of the hospital is maximum at this point.

- $C_{s, i}$ is the cost of storage of drug $i$.

The goals of a pharmacy manager can be summed up in the following list. Note that the goals are provided in decreasing priority:

1) Demand satisfaction. In other words, the probability of drug shortage has to be minimized. The demand of the drugs is non deterministic. The same may happen with the transport delay associated to the shipments. As a consequence, it is common to set a safety stock in order to cope with the uncertainty introduced by these problems. Two possibilities arise at this point depending on whether a fixed or variable safety stock is set up. In the first case, a minimum bound is introduced in the optimization problem. In the second one, the safety stock becomes an optimization parameter. Anyhow, this is translated into the following mathematical condition:

$$
\min _{\delta_{i}^{j}, u_{i}^{j} \forall i, j} \sum_{k=0}^{N} \sum_{i=1}^{N_{i}} C_{o s, i} \operatorname{Pr}\left(s_{i}(t+k)<0\right),
$$

where $\operatorname{Pr}\left(s_{i}(t+k)<0\right)$ stands for the probability of $s_{i}(t+k)$ being negative and $N$ is the length of the time horizon in which the condition has to be satisfied.

2) Minimize the expenses on the acquisition of drugs and 
the inventory levels, that is,

$$
\begin{aligned}
& \min _{\delta_{i}^{j}, u_{i}^{j} \forall i, j} \sum_{k=0}^{N} \sum_{i=1}^{N_{i}} \sum_{j=1}^{n_{p_{i}}} \delta_{i}^{j}(t+k)\left(p_{i}^{j} u_{i}^{j}(t+k)+C_{s h, i}^{j}\right) \\
& +\sum_{k=0}^{N} \sum_{i=1}^{N_{i}} C_{s, i} s_{i}(t+k) .
\end{aligned}
$$

3) Minimize the number of orders placed. The human resources of the pharmacy department are limited. Thus, it is convenient to minimize the fixed costs introduced every time an order is placed. This goal is better understood when it is taken into account that, for example, in a hospital such as Reina Sofía more than twelve thousand orders are placed during a year. Mathematically, this condition is equivalent to the following minimization problem:

$$
\min _{\delta} \sum_{k=0}^{N} \sum_{i=1}^{N_{i}} \sum_{j=1}^{n_{p_{i}}} C_{o p, i} \delta_{i}^{j}(t+k) .
$$

In addition, different constraints have to be taken into account:

- Storage constraints. On one hand, the stock of drug $i$ has to be greater than a safety stock $s_{i, \min }$, whose mission is to provide an extra guarantee so that the probability of lack of inventory is reduced. On the other, there may be room constraints that limit the maximum number of drug samples that can be stored. Therefore,

$$
s_{i} \in\left[s_{i, \min }, s_{i, \max }\right] .
$$

- Order constraints. The constraints on the orders require the use of two different variables. The first one is a boolean variable that represents whether an order of drug $i$ has been placed to provider $j$ during time $t$. Thus, $\delta_{i}^{j}(t) \in[0,1]$. In case of placing an order it has to be taken into account that there is both a minimum and a maximum number of items that can be ordered, that is,

$$
u_{i}^{j} \in\left[u_{i, \min }^{j}, u_{i, \max }^{j}\right] .
$$

- Operational constraints. The pharmacy has a limited capacity for placing orders and receiving shipments. For this reason a limit has to be imposed on the number of orders placed during an horizon of length $N$, that is,

$$
\sum_{k=0}^{N} \sum_{j=1}^{n_{p_{i}}} \delta_{i}^{j}(t+k) \leq \Delta_{i}
$$

where $\Delta_{i}$ is the maximum number of orders of drug $i$ that can be placed during the horizon.

- Economical constraints. We will consider a constraint on the amount of money that can be spent during the horizon $N$, being $\$_{\max }$ the maximum amount. For simplicity, we will ignore the expenses due to the stocked goods. Thus, this goal can be mathematically translated as:

$$
\sum_{\substack{k=0 \\ \leq}}^{N} \sum_{i=1}^{N_{i}} \sum_{j=1}^{n_{p_{i}}} \delta_{i}^{j}(t+k)\left(p_{i}^{j} u_{i}^{j}(t+k)+C_{s h, i}^{j}+C_{o p, i}\right)
$$

\section{Optimization PROBlem AND Model PREDiCTIVE CONTROL IN PHARMACY INVENTORY}

As it was stated in Section II, the objective of the optimization problem is threefold; the demand has to be satisfied, the fixed assets reduced and the number of orders minimized. The system can be represented according to Figure 1.

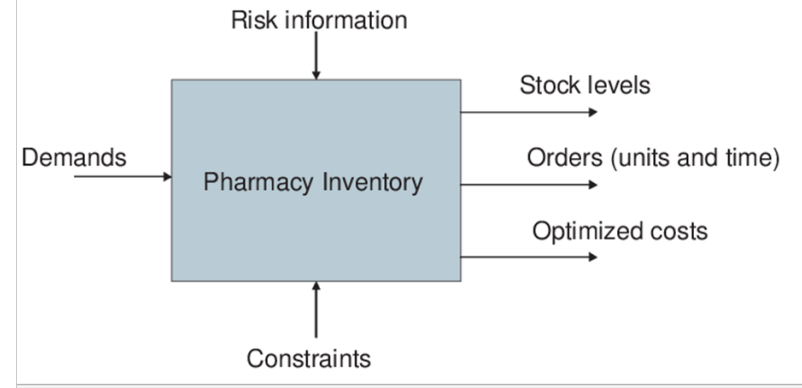

Fig. 1. Block System

System inputs are the estimated drug demands, disturbance and constraints. The outputs are the optimal stock levels, minimum costs and data about when and how many orders should be delivered. The performance index considered in this work involves a multicriteria weighted function where demand satisfaction, expenses and number of orders are included. Note that these terms are defined in Section II as goals of a pharmacy manager, i.e.,

$$
\min _{u} J
$$

$$
\text { with } J=\beta_{1} \Delta(u, t)+\beta_{2} E(u, t)+\beta_{3} \Theta(u, t) \text {, }
$$

where $\Delta, E$ and $\Theta$ are respectively the terms associated to demand satisfaction, costs and orders. Note that the outputs of the problem depend strongly on the weights $\beta$, prioritizing the different terms. These parameters are chosen following the recommendations of the hospital.

At the end, the problem to solve is a deterministic one, with the particularity that the constraints are obtained from probabilistic assumptions.

\section{A. Model Predictive Control}

MPC is a control strategy based on the explicit use of a dynamic model to predict the process output at future time instants over a prediction horizon $(N)$ [3]. The set of future control signals is calculated by optimizing a criterion or objective function. The predicted outputs depend on the known past inputs and outputs values up to instant $k$ and on the future control signals. Only the control signal 
calculated for instant $k$ is sent to the process whilst the next control signals are neglected. Some advantages that MPC presents over other optimization control methods include the relative ease of implementation, the ready extension to the multivariable case, and the natural addition of constraints in the optimization.

In this work, MPC technique has been used to solve the problem. Next, we examine the terms involved in expression (9). The first one is related to the satisfaction of the demand. As it has been said, the demand has a random behavior. Therefore, all we can do is to minimize the probability of drug shortage, as it was shown in expression (2).

\section{B. MPC programming}

In the following, we will present some considerations about the inventory control problem in order to ease its implementation. Hence, the objective function will minimize the number of orders placed and the expenses made. Consider the system defined by:

$$
s(t+1)=s(t)+o(t-\tau)-d(t),
$$

where $s(t)=\left[s_{1}(t), \ldots, s_{N_{i}}(t)\right], d(t)=\left[d_{1}(t), \ldots, d_{N_{i}}(t)\right]$ and $o(t-\tau)=\sum_{j=1}^{n_{p_{i}}} \delta_{i}^{j}\left(t-\tau_{i}^{j}\right) u_{i}^{j}\left(t-\tau_{i}^{j}\right)$ represents the total number of items ordered. As it can be seen, system (10) is equivalent to (1).

The problem to solve is the following:

$$
\min _{o} J
$$

subject to (10) and (5)-(8). In this particular problem, we have to deal with two variables of control: a boolean variable $\delta_{i}^{j}(t)$ and $u_{i}^{j}(t)$, which are components of the control variable $o(t)$. Since finding these two variables together solving the optimization problem is a difficult task, due to the different nature of them, this problem will be solved by means of an exhaustive search algorithm, solving the problem one time for each possible scenario depending on the value of $\left\{\delta_{i}^{j}(t), \ldots, \delta_{i}^{j}(t+N)\right\}$. With this algorithm, the optimization problem is solved with respect to the variable $u_{i}^{j}(t)$.

It is straightforward to see that if $\delta_{i}^{j}(t+k)=0, \quad k \in$ $\{0,1, \ldots, N\}$, the number of ordered items $o_{i}^{j}(t+k)=0$. Therefore, to simplify the problem, the vector of control variables $\left\{u_{i}^{j}(t), \ldots, u_{i}^{j}(t+N)\right\}$ is reduced eliminating the null component $u_{i}^{j}(t+k)$, that is:

$$
\begin{aligned}
& \forall \delta_{i}^{j}(t+k)=0, \quad k \in\{0,1, \ldots, N\}, \\
& \underbrace{\left[\begin{array}{c}
u_{i}^{j}(t) \\
\vdots \\
u_{i}^{j}(t+k) \\
\vdots \\
u_{i}^{j}(t+N)
\end{array}\right]}_{\mathbf{u}_{i}^{j}(t)} \rightarrow \underbrace{\left[\begin{array}{c}
u_{i}^{j}(t) \\
\vdots \\
u_{i}^{j}(t+k-1) \\
u_{i}^{j}(t+k+1) \\
\vdots \\
u_{i}^{j}(t+N)
\end{array}\right]}_{\mathbf{u}_{i}^{j}(t)},
\end{aligned}
$$

where $\mathbf{u}_{i}^{j}(t) \in \mathbb{R}^{N+1}$ and $\mathbf{u}_{i}{ }_{i}^{j}(t) \in \mathbb{R}^{N^{\prime}+1}$, being

$$
N^{\prime}=N-\sum_{k=0}^{N}\left(1-\delta_{i}^{j}(t+k)\right)
$$

Note that this operation, i.e. to reduce the vector $\mathbf{u}_{i}^{j}(t)$ to $\mathbf{u}_{i}^{, j}(t)$, can be achieved by means of a simple change of variable:

$$
\mathbf{u}_{i}^{j}(t)=M \mathbf{u}_{i}{ }_{i}^{j}(t),
$$

where $M \in \mathbb{R}^{N+1} \times \mathbb{R}^{N^{\prime}+1}$.

For example, if $N=3$ :

$$
\mathbf{u}_{i}^{j}(t)=\left[\begin{array}{c}
u_{i}^{j}(t) \\
u_{i}^{j}(t+1) \\
u_{i}^{j}(t+2) \\
u_{i}^{j}(t+3)
\end{array}\right],
$$

and we are assuming these values: $\delta_{i}^{j}(t)=1, \delta_{i}^{j}(t+1)=$ $1, \delta_{i}^{j}(t+2)=0$ and $\delta_{i}^{j}(t+3)=1$. That means that the dimension of $\mathbf{u}_{i}^{j}(t)$ has to be reduced in one order, so $N^{\prime}=2$ and

$$
M=\left[\begin{array}{lll}
1 & 0 & 0 \\
0 & 1 & 0 \\
0 & 0 & 0 \\
0 & 0 & 1
\end{array}\right]
$$

this matrix provides the reduced vector:

$$
\mathbf{u}_{i}^{j}(t)=\left[\begin{array}{c}
u_{i}^{j}(t) \\
u_{i}^{j}(t+1) \\
u_{i}^{j}(t+3)
\end{array}\right] .
$$

Therefore, $\mathbf{u}_{i}^{j}(t)$ contains only the ordered items that are non-zero.

This optimization problem will be solved as many times as possible combinations with the values of $\left\{\delta_{i}^{j}(t), \ldots, \delta_{i}^{j}(t+\right.$ $N)\}$, to avoid this variable in the optimization, so we will obtain the same number of values of the objective function. The optimal combination of the values of $\left\{\delta_{i}^{j}(t), \ldots, \delta_{i}^{j}(t+\right.$ $N)\}$ corresponds with the one that provides the minimal value of the objective function.

It is necessary to pay special attention to the constraints while solving this problem. It is not possible to impose the whole matrix of constraints to the reduced vector $\mathbf{u}_{i}^{j}(t)$, so it is necessary to also apply the change matrix $M$ to the matrix of constraints to impose them only to the control components that we are considering.

\section{C. $C C-M P C$}

In this subsection, the way to treat the constraints stochasticastically is presented. In (10), the aggregate demand $d(t)$ has associated a stochastic disturbance, due to the uncertain nature of $d(t)$. As the state is influenced by additive uncertainties $d(t)$, the constraints can not be represented in a deterministic way. Therefore they are rewritten in a probabilistic manner, e.g.:

$$
P\left(s(t+k) \geq s_{\text {min }}\right) \geq 1-\delta_{s}, \quad \forall k \in\{1, . ., N\},
$$


where $\delta_{s}$ is the probability of failure, so it is the risk bound of stockout. Developing the last expression along the prediction horizon, and assuming that the disturbances behave as a function of a certain probability distribution, it is possible to calculate or estimate the mean and standard deviation of the state variable. For example, for the first instant of the prediction horizon and assuming that the perturbations behave as a normal distribution with mean $\mu$ and standard deviation $\sigma$, i.e., $d(t)=N(\mu, \sigma)$, we get:

$$
P\left(s(t+0)+o(t+0)-d(t+0) \geq s_{\text {min }}\right) \geq 1-\delta_{s},
$$

which can be normalized as follows:

$$
\begin{aligned}
& P\left[\frac{s(t+1)-s(t+0)-o(t+0)-\mu}{\sigma} \geq \frac{s_{\min }-s(t+0)-o(t+0)-\mu}{\sigma}\right] \\
& \geq 1-\delta_{s} \\
& P\left[\frac{s(t+1)-s(t+0)-o(t+0)-\mu}{\sigma} \leq \frac{s_{\min }-s(t+0)-o(t+0)-\mu}{\sigma}\right] \\
& \leq \delta_{s} \\
& \quad \varphi\left(\frac{s_{\min }-s(t+0)-o(t+0)-\mu}{\sigma}\right) \leq \delta_{s} \\
& \quad \frac{s_{\min }-s(t+0)-o(t+0)-\mu}{\sigma} \leq \varphi^{-1}\left(\delta_{s}\right)
\end{aligned}
$$

where $\varphi(\cdot)$ is the probability distribution function. This allows us to write a constraint of the form:

$$
-o(t+0) \leq s(t+0)-s_{\min }+\varphi^{-1}\left(\delta_{s}\right) \sigma+\mu .
$$

Next, for the second time instant in the prediction horizon:

$$
\begin{aligned}
& P\left(s(t+2) \geq s_{\text {min }}\right) \geq 1-\delta_{s} \\
& P\left(s(t+1)+o(t+1)-d(t+1) \geq s_{\text {min }}\right) \geq 1-\delta_{s} \\
& P((s(t+0)+o(t+0)-d(t+0))+o(t+1) \\
& \left.-d(t+1) \geq s_{\text {min }}\right) \geq 1-\delta_{s} \\
& P(s(t+0)+o(t+0)-d(t+0))+o(t+1) \\
& \left.-d(t+1) \geq s_{\text {min }}\right) \geq 1-\delta_{s} .
\end{aligned}
$$

Following the same reasoning of the previous case, we have:

$$
\begin{gathered}
\varphi\left(\frac{s_{\min }-s(t+0)-o(t+0)-o(t+1)-2 \mu}{\sigma \sqrt{2}}\right) \leq \delta_{s} \\
\frac{s_{\text {min }}-s(t+0)-o(t+0)-o(t+1)-2 \mu}{\sigma \sqrt{2}} \leq \varphi^{-1} \delta_{s},
\end{gathered}
$$

which allows us to write the following constraint:

$$
\begin{aligned}
& -o(t+0)-o(t+1) \leq \\
& s(t+0)-s_{\min }+\varphi^{-1} \delta_{s} \cdot \sigma \sqrt{2}+2 \mu .
\end{aligned}
$$

In general, for a prediction horizon $N$, we have the following constraint that has to be included in the optimization problem behind the design of the MPC to implement the chance constraints:

$$
\begin{aligned}
& -\left[\begin{array}{ccccc}
1 & 0 & 0 & \cdots & 0 \\
1 & 1 & 0 & \cdots & 0 \\
1 & 1 & 1 & \cdots & 0 \\
\vdots & & & \vdots \\
1 & 1 & \cdots & 1
\end{array}\right]\left[\begin{array}{c}
o(t+0) \\
o(t+1) \\
o(t+2) \\
\vdots \\
o(t+N-1)
\end{array}\right] \leq\left[\begin{array}{c}
1 \\
1 \\
1 \\
\vdots \\
1
\end{array}\right] s(t+0) \\
& +\left[\begin{array}{ccc}
1 & 1 & 1 \\
1 & 2 & \sqrt{2} \\
1 & 3 & \sqrt{3} \\
\vdots & \vdots & \vdots \\
1 & N & \sqrt{N}
\end{array}\right]\left[\begin{array}{c}
-s_{\min } \\
\mu \\
\varphi^{-1}\left(\delta_{s}\right) \sigma
\end{array}\right]
\end{aligned}
$$

\section{CAse Study and Results}

In this section, we apply the proposed CC-MPC ${ }^{2}$ to one of the most expensive drugs that is used in these hospitals. In addition, this drug deserves special attention since it must be stored in a fridge, which makes even more important to reduce its average stock level. The real name and price of the drug will not be presented in this paper due to confidentiality reasons.

Regarding the controller, a horizon of 8 days has been considered. The evolution of the stock is modeled using the discrete linear model (10). The orders of this drug have a minimum amount of 4 units and the maximum has been set to 1000 . The price of the drug is 250 euros per unit and each order placed implies an additional cost of 2 euros. The deliveries of this drug usually have a delay of 2 days with respect to the moment in which the order was placed. Finally, the demand term of (10) is non deterministic. A probabilistic characterization of its behavior has been calculated for this drug based on historical data. As a result, we have modeled the daily demand as a normal random variable with $\mu=20$ and standard deviation $\sigma=15$.

For simplicity, neither storage cost nor storage limits have been considered at this stage of our work. The only implemented constraint with respect to the stock is that the probability of stockout event has to be lower than 0.001 , i.e., we request a reliability level of $99.999 \%$. This choice for the reliability level is to prioritize the satisfaction of the clinic needs.

A 600 days simulation of the proposed approach is shown in Figure 2. In blue, the evolution of the stock using CC-MPC is shown. In red, the real evolution of the stock according to the hospital data is shown. In both cases, the stock was always positive, but in the case of CC-MPC the average level was 204 units with a standard deviation of 113, which outperforms the results registered by the hospital (a mean of 451 units and a standard deviation of 229). Note that, for the price considered, this difference corresponds to an amount of more than 60000 euros that is invested frozen unnecessarily. Finally, it is also interesting to notice that during the studied period the hospital placed 58 orders while

\footnotetext{
${ }^{2}$ The problem could be solved also using Mixed Integer Techniques but it would imply a much higher computational cost. One of the advantages of CC-MPC is that the computational cost is the same than the one for a classic MPC.
} 

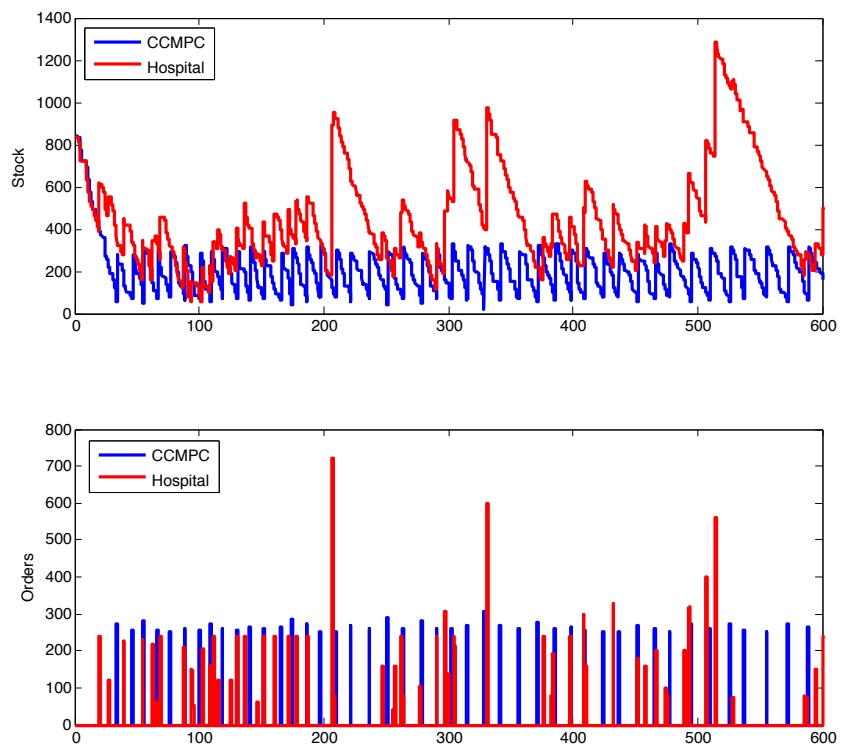

Fig. 2. Real and simulated stock evolution and placed orders.

the CC-MPC placed 44. That is, the CC-MPC obtained better results even with less orders.

The optimization has to be made taking into account the constraints given by (5)-(8). A problem is solved at every sampling time to compute a control sequence $u$ that takes the system to the desired reference. For this drug, the stock reference (security stock) has been set to 2 .

A word of caution has to be said regarding the results at this point: there may be some uncertainty associated with the real data. Sometimes either the drug dispensations or the arrivals of new items are recorded later than they occurred. Another interesting issue regarding the real evolution is its big peaks, which are usually associated to orders placed before holiday periods (no orders can be placed then). In any case, the difference between the reality and the simulation is big enough to believe that the application of this kind of policies in this context is promising.

All optimization problems, solved for the exhaustive algorithm, were computed using a linear programming (linprog in Matlab), on a machine with an Intel Core 2 Duo CPU with $3.33 \mathrm{GHz}$ and $8 \mathrm{~GB}$ RAM.

\section{COnClusions}

In this paper we have described a control-based methodology for decision-making in a pharmacy department to address prevention and control problems in the inventory management. As it can be seen, inventory management is one of the main tasks that a pharmacy department has to carry out in a hospital. It is a complex problem that requires to establish a tradeoff between different and contradictory optimization criteria.

The proposed methodology optimizes the management of the stock while guaranteeing with a very high probability that the drugs will be available for the patients. In this sense, the MPC framework is particularly useful because of its favorable properties, such as ease of constraint-handling.

Finally, it is worthwhile to mention that the proposed technique may provide important economical savings based on the reduction of the average level of stocked drugs while still guaranteeing the satisfaction of the clinical needs of the hospital. Future work will include the extension of the current framework to consider some of the issues that have not been addressed in this paper, like perturbations or time delays, and the real implementation of this control policy into the hospitals that collaborate in this project. It will be interesting also to compare this results with a worst case approach and others probability levels. Finally, a more detailed model of the demand can be developed too.

\section{REFERENCES}

[1] T. Bermejo, B Cuña, V. Napal, and E. Valverde. The hospitalary pharmacy specialist handbook (in Spanish). Spanish Society of Hospitalary Pharmacy, 1999.

[2] A. M. Brewer, K. J. Button, and D. A. Hensher. Handbook of logistics and supply-chain management. Pergamon, 2001.

[3] E. F. Camacho and C. Bordons. Model Predictive Control in the Process Industry. Second Edition. Springer-Verlag, London, England, 2004

[4] A. Geletu, M. Klöppel, H. Zhang, and P. Li. Advances and applications of chance-constrained approaches to systems optimisation under uncertainty. International Journal of Systems Science, 44(7):1209-1232, 2013.

[5] J.M. Grosso, C. Ocampo-Martinez, V. Puig, and B. Joseph. Chanceconstrained model predictive control for drinking water networks. Journal of Process Control, 2014. In press.

[6] P. Kall and J. Mayer. Stochastic linear programming. Springer, New York, NY, 2005.

[7] J. M. Maestre, David Muñoz de la Peña, and E. F. Camacho Distributed model predictive control based on a cooperative game. Optimal Control Applications and Methods, 2010. In press.

[8] A. Prekopa. On probabilistic constrained programming. In In Proceedings of the Princeton Symposium on Mathematical Programming, pages 113-138, Princeton University Press, 1970.

[9] H. Rasku, J. Rantala, and H. Koivisto. Model reference control in inventory and supply chain management. In First International Conference on Informatics in Control, Automation and Robotics, 2004.

[10] A.T. Schwarm and M. Nikolaou. Chance-constrained model predictive control. AIChE Journal, 45(8):1743-1752, 1999.

[11] C. Stoica, M.R. Arahal, D.E. Rivera, and D. Rodríguez-Ayerbe, P.and Dumur. Application of robustified model predictive control to a production-inventory system. In Proceedings of the 48th conferece on decision and control, 2009.

[12] S. Tayur, R. Ganeshan, and M. Magazine. Quantitative models for supply chain management. Kluwer Academic Publisher, 1999.

[13] K. G. Kempft W. Wang, D. E. Rivera and K. D. Smith. A model predictive control strategy for supply chain management in semiconductor manufacturing under uncertainty. In Proceeding of the 2004 American Control Conference, 2004.

[14] Wenlin Wang, Daniel E. Rivera, and Karl G. Kempf. A novel model predictive control algorithm for supply chain management in semiconductor manufacturing. In Proc. of the American Control Conference, 2005. 